\title{
BMJ Open MyCare study: protocol for a controlled trial evaluating the effect of a community-based intervention on psychosocial, clinical outcomes and hospital admission rates for adults with severe mental illness
}

\author{
Renée O'Donnell (D) , ${ }^{1}$ Melissa Savaglio, ${ }^{1}$ Debra Fast, ${ }^{2}$ Ash Vincent, ${ }^{2}$ Dave Vicary, ${ }^{3}$
} Helen Skouteris ${ }^{1,4}$

To cite: 0'Donnell $\mathrm{R}$, Savaglio M, Fast D, et al. MyCare study: protocol for a controlled trial evaluating the effect of a community-based intervention on psychosocial, clinical outcomes and hospital admission rates for adults with severe mental illness. BMJ Open 2020;10:e040610. doi:10.1136/ bmjopen-2020-040610

- Prepublication history and additional material for this paper is available online. To view these files, please visit the journal online (http://dx.doi.org/10. 1136/bmjopen-2020-040610).

Received 18 May 2020 Revised 23 September 2020 Accepted 17 0ctober 2020

Check for updates

(C) Author(s) (or their employer(s)) 2020. Re-use permitted under CC BY-NC. No commercial re-use. See rights and permissions. Published by BMJ.

For numbered affiliations see end of article.

Correspondence to Professor Helen Skouteris; helen.skouteris@monash.edu

\section{ABSTRACT}

Introduction People with serious mental illness (SMI) often fail to receive adequate treatment. To provide a higher level of support, mental health systems have been reformed substantially to integrate mental healthcare into the community. MyCare is one such community-based mental health model of care. This paper describes the study protocol of a controlled trial examining the effect of MyCare on psychosocial and clinical outcomes and hospital admission and duration rates for adults with SMI.

Methods and analysis This is a multisite nonrandomised controlled trial with a 3, 6 and 12-month follow-up period. The study participants will be adults (18-64 years of age) with SMI recruited from Hobart, Launceston and the North-West of Tasmania. The treatment group will include adults who receive both the MyCare intervention and standard mental health support; the control group will include adults who receive only standard mental health support. The primary outcome includes psychosocial and clinical functioning and the secondary outcome will examine hospital admission rates and duration of stay. Mixed-effects models will be used to examine outcome improvements between intake and follow-up. This trial will generate the evidence needed to evaluate the effect of a community mental health support programme delivered in Tasmania, Australia. If MyCare results in sustained positive outcomes for adults with SMI, it could potentially be scaled up more broadly across Australia, addressing the inequity and lack of comprehensive treatment that many individuals with SMI experience.

Ethics and dissemination This study has been approved by the Tasmanian Health and Medical Human Research Ethics Committee. The findings will be disseminated to participants and staff who delivered the intervention, submitted for publication in a peer-reviewed journal and shared at academic conferences.

Trial registration number ACTRN12620000673943.
Strengths and limitations of this study

- This research will employ a longitudinal controlled design which will aid in demonstrating the extent to which MyCare is effective in addressing the psychosocial and clinical needs of individuals with serious mental illness (SMI).

- The treatment group will receive standard mental health support in conjunction with MyCare, to make casual inferences about the effect of the programme when all other treatment is held constant.

- The extended follow-up period postintervention (ie, up to 12 months postclosure) will help to determine whether changes in outcomes among individuals with SMI can be sustained following engagement in MyCare.

- Blinding clinicians and participants to group allocation is not possible as it would be both impractical and unethical.

- There may be unintended significant systematic differences between the two groups due to the nature of group allocation, however, these differences will be minimised as the eligibility criteria are the same for both groups and they can be controlled for within the data analysis.

\section{INTRODUCTION}

\section{Background}

Serious mental illness (SMI), defined as a mental, behavioural or emotional disorder that can have episodic, recurrent or persistent features resulting in distress and functional impairment ${ }^{1}$, is a leading cause of disability around the world. ${ }^{2}$ Persons with SMI often fail to receive adequate treatment; currently it is estimated that only one in five people with SMI receive sufficient treatment to address their clinical needs. ${ }^{3-6}$ Issues pertinent at the system (ie, service shortage) and individual 
(ie, stigma) level contribute significantly to this high prevalence of inadequate treatment. ${ }^{47}$

To address this, mental health systems have undergone substantial reforms to integrate mental health services (MHS) into the community. ${ }^{89}$ Indeed, it has been established that mental healthcare delivered in the community has the potential to address system-level barriers that contribute to high rates of untreated SMI. ${ }^{10}$ The most prominent and universal community treatment model offered to individuals with SMI is Assertive Community Treatment (ACT). ACT is an intensive and long-term assertive outreach model in which a multidisciplinary team of healthcare professionals deliver integrated services to clients, including regular home visits from case workers, housing and employment support, drug and alcohol services, case management and counselling. ${ }^{11}$ With the programme's multidisciplinary team approach, wrap-around support on a needs-led basis, and long-term duration, there is a strong evidence base for its success in reducing hospitalizations and improving psychosocial functioning among those with SMI. ${ }^{12-14}$ However, ACT is limited by its strict eligibility criteria (ie, often excludes those with a primary diagnosis of substance misuse problems and personality disorders ${ }^{15}$ ). Moreover, at the point of closure from ACT, there is little effort directed to transitioning the person to stepdown support. ${ }^{16} 17$ Consequently, clients have reported feeling isolated and alone at the point of closure from ACT, ${ }^{18}$ impeding on their recovery and increasing chances of readmission into acute care. In the Australian community mental health sector, ACT is a less-established model of care as only few studies have evaluated its effectiveness and obtained promising findings.

To address the shortcomings of ACT and the increased demand of support needed for persons with SMI, funding was provided to an Australian Community Service Organisation to develop MyCare. MyCare, informed by the successful principles of ACT (ie, assertive outreach approach, provision of wrap-around services and support, and extensive duration), is delivered in conjunction with MHS and aims to provide assertive outreach support with a focus on promoting psychosocial health and reducing clinical symptomology. The eligibility criteria of MyCare is more flexible than that provided by ACT programmes (ie, acceptance of persons with personality disorders and/or substance misuse concerns). Furthermore, at the point of closure, MyCare clients are integrated into less intensive services to ensure their recovery is ongoing. A table outlining the differences between MyCare and ACT can be found in part A of online supplemental material.

A preliminary pilot evaluation of MyCare was completed in 2019. ${ }^{19}$ The results showed the programme was successfully implemented-yet the extent to which the programme was effective in improving client outcomes was not examined. To address this, the aim of the current study is to use a longitudinal non-randomised controlled trial to examine the effectiveness of MyCare in improving client outcomes, as compared with those who receive standard MHS treatment.

\section{Current study}

The aim of this study is to examine the effect of MyCare on psychosocial and clinical outcomes and hospital admission and duration rates for adults with SMI, compared with adults with SMI who receive standard MHS. Participants in the treatment group will receive MHS in conjunction with MyCare. Therefore, using MHS as a comparison group was chosen to make casual inferences of the effect of the MyCare programme, when all other treatment is held constant. The current study is critical for generating Australian evidence regarding the effectiveness of a community-based mental health programme that aims to provide assertive outreach support to individuals with SMI. Importantly, if the programme is found to yield significant improvements in outcomes, then this would provide strong support for the scale-up and dissemination of MyCare across Australia. In turn, this would begin to address the inequity and lack of comprehensive treatment and that many individuals with SMI currently face.

\section{METHODS AND ANALYSIS}

\section{Study design and setting}

This is a longitudinal non-randomised controlled comparison study that will be conducted across multiple sites in Hobart, Launceston and the NorthWest of Tasmania, Australia. Participants will be recruited from the local MHS at each site. The study design is depicted in figure 1. After completion of a baseline assessment, recruited participants will be assigned to either the MyCare treatment group or the MHS control group. Group allocation will be alternated such that the first 20 participants are assigned to MyCare, followed by 20 to MHS. This process of group assignment will continue until each group has

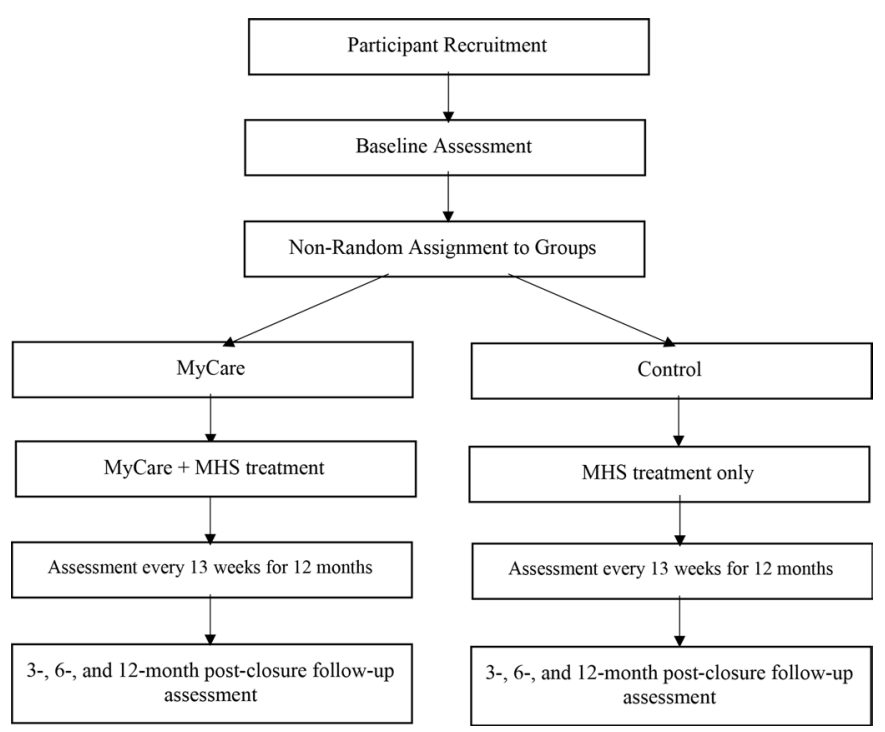

Figure 1 Visual Depiction of Mycare trial study design. MHS, mental health services. 
100 participants as this is the capacity of MyCare. It is anticipated that it will take a period of 5 months to reach this quota. Recruitment will commence in October 2020.

\section{Participants}

\section{Inclusion and exclusion criteria}

Participants will be referred into the programme through their local MHS. Participants will be eligible for this trial if they are aged 18-64 years and have a diagnosis of an SMI, which will encompass the following: schizophrenia and other related psychotic disorders, personality disorders and mood disorders (eg, depressive or bipolarrelated disorders) categorised as 'severe' according to the Diagnostic and Statistical Manual of Mental Disorders. ${ }^{20}$ These disorders are categorised by significant clinical and functional impairment, requiring intensive multidisciplinary care and psychosocial support. ${ }^{20}$ It is expected that the proportion of participants with each type of SMI will align with the local population prevalence of such diagnoses. Additionally, participants will be included if they can speak and understand English, and if they reside in Hobart, Launceston, or the North-West of Tasmania. Individuals who are not actively case managed by MHS will be excluded. Moreover, participants who are engaged in any other type of intervention support for their mental health will be excluded from the study.

\section{Sample size calculation}

Sample size calculations were conducted using G*Power. ${ }^{21}$ Systematic reviews of comparable interventions ${ }^{21}{ }^{22}$ have found small to medium effects for post intervention outcomes including the primary outcome of psychosocial and clinical health and the secondary outcome of hospital admission rate and duration of stay. With the alpha set at 0.05 , correlations between repeated measures set at 0.50 and power of 0.80 , a total sample of 165 individuals will be required (across both groups). However, taking into account $20 \%$ attrition that is typical of these studies, ${ }^{22} 200$ participants will be recruited to obtain a final sample size of at least 165 participants.

\section{Intervention}

Participants in the treatment group will receive support from their MyCare Care Coordinator and their MHS Case Manager for 12 months.

\section{Intervention content}

At the point of referral into the programme via MHS, a MyCare Senior Practitioner will assess the eligibility of the individual for MyCare. If eligible, MyCare participants will then be allocated a MyCare Care Coordinator who provides home visitations on either a twice-weekly, weekly or fortnightly basis (COVID-19 will likely influence the delivery of frequent face-to-face home visitations. Therefore, contact may alternate between face-to-face and virtual meetings), depending on their needs. The aim of the home visitations is to assist individuals in setting and achieving their own personalised psychosocial goals and to reduce clinical symptomology (eg, Care Coordinators may accompany participants while grocery shopping to assist in improving eating habits). MyCare also incorporates a brokerage component, which can be used to assist in funding activities to help participants obtain psychosocial goals (ie, gym membership) or to provide them with basic necessities to assist with symptom management (ie, purchasing medication). Prior to closure, MyCare Care Coordinators gradually reduce their intensity and frequency of support and seek to refer participants to less intensive community services as needed. After 12 months, Care Coordinators can provide telephone follow-up for up to 3 months to facilitate smooth transition from MyCare to other services. The support offered by MyCare is complemented by participants' existing MHS Case Worker who will manage the clinical treatment of their mental illness. Therefore, the MHS case manager will be the primary coordinator of participants' mental healthcare. The role of MHS is described in more detail below in the control section. A table outlining the differences between MyCare and MHS can be found in part B of online supplemental material.

\section{Intervention implementation}

MyCare will be implemented through a partnership between the MyCare Care Coordinators and MHS Case Managers who will work closely together to capture and share local knowledge regarding each participant's progress. In this approach, it is proposed that continual monitoring and review of participants' clinical and psychosocial outcomes will be achieved. MyCare Care Coordinators are trained mental health practitioners, including psychologists, social workers and counsellors, with a wealth of experience providing care to individuals with an SMI. In addition to the provision of psychosocial support, care coordinators can assist with medication adherence (ie, reminding participants to take medication, encouraging adherence, providing psychoeducation), monitoring symptoms, and identifying signs of relapse or significant decline in functioning. In doing so, they will provide feedback to the participants' MHS case manager accordingly, demonstrating the importance of the collaborative partnership and regular contact between MyCare and MHS. To facilitate engagement and adherence, the MyCare Care Coordinator will dedicate the first 3 months of the programme towards building a strong relationship with the participant. The MyCare Care Coordinator will continue to attempt engagement even when participants appear unmotivated, resistant or isolate themselves. Additionally, the 6:1 client to staff caseload will permit the MyCare Care Coordinators to adopt a tailored, personalised approach and operate on a needs-led basis depending on each person's required level of support. The smaller caseloads seek to promote adaptability and flexibility, prolonged support, and strengthened relationships to maintain engagement and facilitate positive outcomes among participants. 


\section{Control}

The control group will be comprised of voluntary participants who are only engaged with MHS. They will receive support via their MHS Case Manager for 12 months. MHS is operated through the Tasmanian Department of Health, and will provide individuals with ongoing clinical assessment, treatment, education and support to manage their SMI and promote clinical improvements. MHS focuses solely on addressing the clinical symptoms of the mental illness, whereas the MyCare programme seeks to facilitate the achievement of participants' psychosocial goals in the community. MHS case managers are embedded within a team of clinical psychologists, psychiatrists, mental health nurses and other specialist medical staff to provide mental health clinical support. MHS case managers have a caseload of approximately 25 clients each, and provide ongoing assessment, psychoeducation, symptom management, monitor and support medication adherence, administer medication where necessary, and facilitate participant's engagement with psychology where needed.

Participants' frequency of contact with MHS will be fortnightly to monthly, comprising of in-person visits to the local outpatient clinic or telephone support. Home visits or other forms of assertive outreach are not part of standard care, and there is less focus on engagement and rapport building than MyCare. MHS case managers will often refer clients to other community-based services, yet there is strictly no telephone follow-up or contact permitted postclosure between MHS case managers and clients. All participants will continue with their existing MHS case manager during the trial period, regardless of whether they are allocated to the treatment or control condition. Individuals who are engaged with MHS and other community-based programmes will not be eligible for the control group due to potential contamination of effects.

\section{Patient and public involvement}

Patient and public involvement for this study protocol occurred through a consumer-carer advisory group (ie, a group that includes existing clients, carers and staff from both MyCare and MHS). This group partnered with us for the design of the study, advocating for a longer follow-up period, assessment time points that coincided with the programmes' existing reviews, and the necessity for clients to have a consistent staff member complete their assessments throughout the study period. They also provided guidance and input regarding the assessment material to support the intervention and we assessed the burden of the intervention from the clients' perspective. This advisory group will assist with the dissemination plan, and all participants will be provided with a copy of the findings.

Throughout the trial, this advisory group will meet monthly to discuss their experiences of implementing and receiving MyCare and MHS, and the key components of the study. Following group allocation, additional representative clients from both the intervention and control groups, as identified by their MyCare Coordinator or MHS case manager, will be included in this advisory group. Specifically, the role of this advisory group will be to provide feedback about their experiences of incorporating the study components into the ongoing implementation of the intervention and control groups (ie, what is working well, what is not working well), how to ensure that the implementation of MyCare continues to meet the needs of the end-user, and to discuss their experiences of completing the assessments (ie, barriers to completing assessments, clarifying both staff's and clients' understanding of items). Changes to the studyrelated methods may be made throughout the trial if warranted based on the ongoing feedback from the advisory group. If this is deemed necessary, relevant parties will be notified. The content of the monthly meetings will be predominantly study related; clinical care and input will not be the focus of these sessions. Therefore, participants' confidentiality will be protected as the group will not be discussing individuals' clinical care, progress or functioning.

\section{Data collection}

The following data will be collected from all participants: (1) basic demographic information, (2) psychosocial outcomes (ie, Life Skills Profile 16 (LSP-16), Behavioural and Symptom Identification Scale 32 (BASIS-32)), (3) clinical outcomes (ie, Health of the Nations Outcome Scales (HoNOS)) and (4) hospital admission rates and duration of stay (refer to part C of online supplemental material for more information). Data will be collected at baseline (intake), every 13 weeks during the programme (interim), at 12 months (closure) and at 15, 18 and 24 months follow-up (ie, 3, 6 and 12 months postclosure). Psychosocial and clinical data will be collected by the MyCare Care Coordinator for the treatment group and MHS Case Manager for the control group. Hospital data will be reported by the Tasmanian Health Service. Where possible, all of participants' data, including postclosure assessments, will be collected by the same person (ie, their MyCare Care Coordinator or MHS Case Manager, depending on group assignment) throughout their involvement in this trial. All MyCare Care Coordinators and MHS case managers participating in the trial will be trained in administering the three rating scales by the principal researcher to establish inter-rater reliability. Additionally, all staff will receive a manual to guide the administration of each scale, which will include definitions for each item (ie, definitions for 'problem drinking', 'cognitive problems', etc), to reduce variability associated with individual interpretation. Please note that the MyCare Care Coordinators (for the treatment group) and MHS Case Managers (for the control group) will obtain participants' informed consent before data collection commences (see part D of online supplemental material). 


\section{Outcome measures}

\section{Psychosocial outcomes}

The BASIS-32 is a 32-item psychosocial questionnaire that assesses the extent of difficulty that an individual is experiencing on a range of psychosocial functioning areas (eg, self-confidence and managing day-to-day life). Each item is scored on a 5-point Likert scale of 0 (no difficulty) to 4 (extreme difficulty). The higher the score, the greater the level of functional difficulty that the client is experiencing. The BASIS-32 demonstrates high internal reliability ( $\mathrm{a}=0.75$ to 0.91 ) and validity. ${ }^{23} 24$

The LSP-16 is a 16-item psychosocial questionnaire used to assess a person's basic life skills (eg, maintaining a healthy diet and medication adherence). The scale is rated on a 4-point Likert scale from 0 (no difficulty) to 3 (extreme difficulty). The higher the score, the greater the difficulty that the client is experiencing in their general functioning and life skills. The LSP-16 exhibits moderate to high reliability $(\mathrm{a}=0.67-0.88)$ and validity. ${ }^{25} 26$

\section{Clinical outcomes}

The HoNOS is a 12-item questionnaire that assesses clinical problems across four domains: (1) behaviour (ie, overactive, aggressive, problem drinking, drug taking, selfinjury); (2) impairment (ie, cognitive problems, physical disability); (3) symptoms (presence of mental disorder) and 4) social functioning (ie, social relationships, daily activities, living conditions, employment). Each item is rated on a 5-point Likert scale of 0 (no problem) to 4 (severe problem). The individual receives a total score for each of the four domains, as well as an overall score. The higher the score, the greater the severity of problems that the client is experiencing within the four domains. The HoNOS is well regarded as an appropriate clinical tool with moderate to high internal consistency $(\mathrm{a}=0.59-0.76)$ and validity. ${ }^{27}$

\section{Hospital admission/s and length of stay}

Data regarding hospital admission/s and duration of stay (ie, days in hospital) will be collated and reported by Tasmanian Health Service in Hobart and Launceston. Specifically, date of hospital admission, reason for admission, diagnosis, days in hospital, and date of discharge will be collected for hospitalisations during the trial period and the prior 2 years. Refer to part $\mathrm{C}$ of online supplemental materials for more information.

\section{Data analyses}

The primary outcome of this study is to examine the degree to which there are improvements in psychosocial and clinical indicators and the secondary outcome will examine hospital admission rates and duration of stay. A total of four mixed-effects models will be run to examine these outcomes. The models will be run including fixed and random effects. For the dichotomous variables (ie, hospital admission/s) a logistic mixed-effects model will be run to examine if there is a change in the outcomes over time. For the continuous variables (ie, hospital duration of stay, clinical and psychosocial outcomes), a binomial or Poisson mixed-effects model will be run depending on the distribution of the data and how skewed it is. Please note that missing data does not undermine the application of mixed-effects analyses as it applies maximum likelihood estimation (as opposed to analysis of variance that employs listwise deletion for missing data). Cohen's $\mathrm{d}$ effect size benchmarks of $0.20,0.50$ and 0.80 indicating small, moderate and large effects, respectively, will be used to determine minimum clinically important differences between groups. ${ }^{28}$ Interim analyses will be conducted to obtain preliminary findings.

\section{ETHICS AND DISSEMINATION \\ Data management}

The data collected will be managed by the programme manager from each programme (ie, MyCare and MHS). The managers are trained on how to deidentify the information (ie, remove the name of the participant and replace with a unique identification code). Thus, all participants will be provided with a unique numerical identifier for use throughout the study, permitting participant progress to be tracked, anonymously, throughout the duration of the study. A secure, password-protected online database will be used to record and store all data and only one member from the research team will be permitted access to this. At the conclusion of this study, the chief investigators from Monash University and Baptcare will have access to the final dataset. This study has been approved by the Tasmanian Health and Medical Human Research Ethics Committee (Project Number BLINDED). Project materials will be stored for a period of 6 years. Thereafter, hard copy documents and online files will be deleted. Any adverse events that occur as a result of this study will be reported to the Tasmanian Health and Medical Human Research Ethics Committee. An independent audit will occur once a year to ensure that the data being collected and stored is accurate and free from error.

\section{Dissemination}

The findings of this study will be disseminated to participants and the staff who were involved in the delivery of MyCare. The findings are also planned to be published in a peer-reviewed journal and presented at academic conferences. Moreover, any changes made to this protocol will be communicated to relevant parties.

\section{Author affiliations \\ ${ }^{1}$ Monash Centre for Health Research and Implementation, Monash University, Clayton, Victoria, Australia \\ ${ }^{2}$ Baptcare, Tasmania, Victoria, Australia \\ ${ }^{3}$ Baptcare, Footscray, Victoria, Australia \\ ${ }^{4}$ Warwick Business School, University of Warwick, Coventry, UK}

Contributors $\mathrm{RO}$ and MS were responsible for the write up of this study protocol. $\mathrm{HS}, \mathrm{DF}, \mathrm{AV}$ and DV were responsible for the development of the study design and revising manuscript drafts.

Funding This research has been funded by Baptcare and sponsored by Monash University. 
Competing interests None declared.

Patient consent for publication Not required.

Provenance and peer review Not commissioned; externally peer reviewed.

Supplemental material This content has been supplied by the author(s). It has not been vetted by BMJ Publishing Group Limited (BMJ) and may not have been peer-reviewed. Any opinions or recommendations discussed are solely those of the author(s) and are not endorsed by BMJ. BMJ disclaims all liability and responsibility arising from any reliance placed on the content. Where the content includes any translated material, BMJ does not warrant the accuracy and reliability of the translations (including but not limited to local regulations, clinical guidelines, terminology, drug names and drug dosages), and is not responsible for any error and/or omissions arising from translation and adaptation or otherwise.

Open access This is an open access article distributed in accordance with the Creative Commons Attribution Non Commercial (CC BY-NC 4.0) license, which permits others to distribute, remix, adapt, build upon this work non-commercially, and license their derivative works on different terms, provided the original work is properly cited, appropriate credit is given, any changes made indicated, and the use is non-commercial. See: http://creativecommons.org/licenses/by-nc/4.0/.

ORCID iD

Renée 0'Donnell http://orcid.org/0000-0003-2563-2867

\section{REFERENCES}

1 Baker J, Travers JL, Buschman P, et al. An efficient nurse-led community-based service model for delivering coordinated care to persons with serious mental illness at risk for homelessness. J Am Psychiatr Nurses Assoc 2017;24:1-8.

2 World Health Organisation [WHO]. Mental health action plan 20132020. Geneva: Switzerland: WHO Document Production Services, 2013.

3 Australian Bureau of Statistics. National survey of mental health and wellbeing: summary of results, 2007. Available: http://www. abs.gov.au/ausstats/abs@.nsf/Latestproducts/4326.0Media\% 20Release12007?opendocument\&tabname=Summary\&prodno= 4326.0\&issue $=2007 \&$ num $=\& v i$ [Accessed Apr 2020].

4 Kessler RC, Berglund PA, Bruce ML, et al. The prevalence and correlates of untreated serious mental illness. Health Serv Res 2001;36:987-1007.

5 Lund C, Breen A, Flisher AJ, et al. Poverty and common mental disorders in low and middle income countries: a systematic review. Soc Sci Med 2010;71:517-28.

6 McManus S, Meltzer H, Brugha TS, et al. Adult psychiatric morbidity in England, 2007: results of a household survey. The NHS information centre for health and social care, 2009. Available: https://digital.nhs. uk/data-and-information/publications/statistical/adult-psychiatricmorbidity-survey/adult-psychiatric-morbidity-in-england-2007results-of-a-household-survey

7 Corrigan P. How stigma interferes with mental health care. Am Psychol 2004;59:614-25.

8 Griffiths KM, Mendoza J, Carron-Arthur B. Where to mental health reform in Australia: is anyone listening to our independent auditors? Med J Aust 2015;202:172-4.

9 Whiteford HA, Buckingham WJ. Ten years of mental health service reform in Australia: are we getting it right? Med J Aust 2005;182:396-400.
10 Grazier KL, Smiley ML, Bondalapati KS. Overcoming barriers to integrating behavioral health and primary care services. J Prim Care Community Health 2016;7:242-8.

11 Clausen H, Ruud T, Odden S, et al. Hospitalisation of severely mentally ill patients with and without problematic substance use before and during Assertive community treatment: an observational cohort study. BMC Psychiatry 2016;16:125-35.

12 Bond G, Drake RE, Mueser KT, et al. Assertive community treatment for persons with severe mental illness: critical ingredients and impact on patients. Disease Management and Health Outcomes 2001;9:141-59.

13 Drukker M, Visser E, Sytema S, et al. Flexible assertive community treatment, severity of symptoms and psychiatric health service use, a real life observational study. CPEMH 2013;9:202-9.

14 Marshall M, Lockwood A, Cochrane Schizophrenia Group. Assertive community treatment for people with severe mental disorders. Cochrane Database Syst Rev 2011;46.

15 Reynolds SK, Wolbert R, Abney-Cunningham G, et al. Dialectical behavior therapy for assertive community treatment teams. In: Dimeff L, Koerner K, eds. Dialectical behavior therapy in clinical practice: applications across disorders and settings. New York: Guilford Press, 2007.

16 Bromley E, Mikesell L, Whelan F, et al. Clinical factors associated with successful discharge from assertive community treatment. Community Ment Health J 2017;53:916-21. 2017.

17 Finnerty MT, Manuel Jl, Tochterman AZ, et al. Clinicians' perceptions of challenges and strategies of transition from assertive community treatment to less intensive services. Community Ment Health J 2015;51:85-95

18 Chen F-P, Herman DB. Discharge practices in a time-unlimited intervention: the perspectives of practitioners in assertive community treatment. Adm Policy Ment Health 2012;39:170-9.

19 O’Donnell R, Ayton D, Pizzirani B, et al. MyCare - enhancing community mental health care in Tasmania, Australia: program description and implementation findings. Australian Journal of Primary Health 2020;26:18-25.

20 American Psychiatric Association. Diagnostic and statistical manual of mental disorders. Fifth edition. Washington: American Psychiatric Association, 2013

21 Faul F, Erdfelder E, Lang A-G, et al. G*Power 3: a flexible statistical power analysis program for the social, behavioral, and biomedical sciences. Behav Res Methods 2007;39:175-91.

22 Leamy M, Bird V, Le Boutillier C, et al. Conceptual framework for personal recovery in mental health: systematic review and narrative synthesis. Br J Psychiatry 2011;199:445-52.

23 Cameron IM, Cunningham L, Crawford JR, et al. Psychometric properties of the BASIS-24C (behaviour and symptom identification Scale-Revised) mental health outcome measure. Int J Psychiatry Clin Pract 2007;11:36-43.

24 Uttaro T, González A. Psychometric properties of the behavior and symptom identification scale administered in a crisis residential mental health treatment setting. Psychol Rep 2002;91:439-43.

25 Parker G, Rosen A, Emdur N, et al. The life skills profile: psychometric properties of a measure assessing function and disability in schizophrenia. Acta Psychiatr Scand 1991;83:145-52.

26 Rosen A, Hadzi-Pavlovic D, Parker G. The life skills profile: a measure assessing function and disability in schizophrenia. Schizophr Bull 1989;15:325-37.

27 Pirkis JE, Burgess PM, Kirk PK, et al. A review of the psychometric properties of the health of the nation outcome scales (HoNOS) family of measures. Health Qual Life Outcomes 2005;3:76-12.

28 Copay AG, Subach BR, Glassman SD, et al. Understanding the minimum clinically important difference: a review of concepts and methods. Spine J 2007;7:541-6. 